\title{
Anatomical study of supraclavicular perforator artery and its clinical application as sensate supraclavicular artery propeller flap in the reconstruction of post burns scar contracture neck
}

\author{
T.M. Balakrishnan ${ }^{1}$ and N. Sivarajan ${ }^{2}$ \\ ${ }^{1}$ Plastic surgery, Govt.General Hospital, Chennai-600003, India \\ ${ }^{2}$ General Surgical Department, Chettinad Hospital and Research Institute, Rajiv Gandhi salai, Kelambakkam, \\ Kancheepuram Dt-603 103, Chennai, India \\ sivarajandr@yahoo.com
}

\begin{abstract}
Post burns scar contracture of neck has severe functional and aesthetic considerations. The mortality and morbidity from burns has reduced considerably for the last six decades does not truly reflect whether the victim can lead a normal life, as the head and neck region is an aesthetically demanding area to resurface because of high visibility. The use of supraclavicular perforator artery flap is a well described and established technique. In our study after understanding intricacy of the supraclavicular artery perforator anatomy as it is used as sensate pedicled flap for reconstruction of post burns contracture of neck, we have established that sensation from middle supraclavicular nerve represents an extremely versatile and useful option for resurfacing post burns scar contracture of neck. Anatomical study of 10 fresh cadavers in which 20 transverse cervical vascular system traced up to the thyrocervical trunk and various findings noted and applied clinically on 16 patients, we conclude supraclavicular artery propeller flap provides good aesthetic and functional results.
\end{abstract}

Keywords: Supraclavicular perforator artery, Sensate flap, Post burns scar contracture of neck.

\section{Introduction}

The cervical region is functionally and anatomically designed to achieve a maximum range in threedimensional motion. The visibility of this area poses a special challenge for the surgeon in giving an aesthetically satisfactory result. Mentosternal contractures are well-known complications after burns of any etiology. These have great physical and psychological impact on the patient. Moreover, these contractures may exert traction forces that may pull the lower lip, chin, cheek and lower eye lid caudally. The various reconstructive options available for resurfacing of this region range from split thickness skin grafts and ultrathin flaps to local or free flaps. For the face, one has to take account of the aesthetic units and provide an appropriately thin flap to restore both form and function. The colour and texture match is also equally important. The commonly done procedure of release of these contractures and split-skin grafting suffers on all these counts. Again, the cervico-mental angle deserves special attention for functional and aesthetic reasons. A locoregional flap should also leave a minimum of donor site morbidity and preferably be hidden beneath the clothing. Hence, the more adjacent the donor site, with better colour, thickness, texture match and intact sensation offers best reconstruction (Achauer, 1991).

In our experience, the supraclavicular perforator artery-based flap with sensory supply from middle supra clavicular nerve is a logical choice for head and neck resurfacing, offering advantages of good color and texture match, with proprioception established at the reconstructed site facilitates rapid functional recovery. An added advantage is a concealed donor site by Indian type of clothing especially in females. The chance of recurrence of the contracture as seen with split skin grafts is also absent and post operative splinting is not used in our cases. We studied the Anatomical variations of supraclavicular perforator artery on cadavers and traced up to the thyrocervical trunk and it's variations noted and clinically applied that knowledge on mentosternal post burns scar contracture of neck in restoration of an aesthetically acceptable and a functional neck.

Materials and method

Anatomic study (Period of study-March2010 to September 2010)

10 fresh cadavers and therefore 20 transverse cervical vascular system were examined up to thyrocervical Trunk. Dissection and measurement of dimensions using calipers and measuring tapes were used in our study. Supraclavicular perforator is identified by combination of anterograde and retrograde dissection by starting the dissection with infra clavicular incision. Transverse cervical artery in all specimens examined is arising separately from trifurcation of thyrocervical trunk which in turn is the branch from first part of subclavian artery. In all specimens the supraclavicular artery arises from the Thyrocervical artery as it crosses upper trunk on an average $8.5 \mathrm{cms}$ from medial end of clavicle and 7.5 cms from mid point of clavicle with $1.5 \mathrm{~mm}$ average diameter at its origin. Significant variation noted in our study is that it is not accompanied by any venae comitantes and it is single right from its origin and it is always lateral to triangle which was bound medially Sternocleidomastoid muscle, laterally external jugular vein and inferiorly clavicle which is described for its identification in literature so far as passing through this triangle (Pallua et al., 1997, Cormack et al.,1983, 1984) and also by dissection we can render a less than $2 \mathrm{~cm}$ diameter neurovascular hilum where External jugular vein, mid supraclavicular nerve and supraclavicular 
Fig. 1. Facio cutaneous dissection showing supraclavicular Artery perforator without any venaecommitantes.

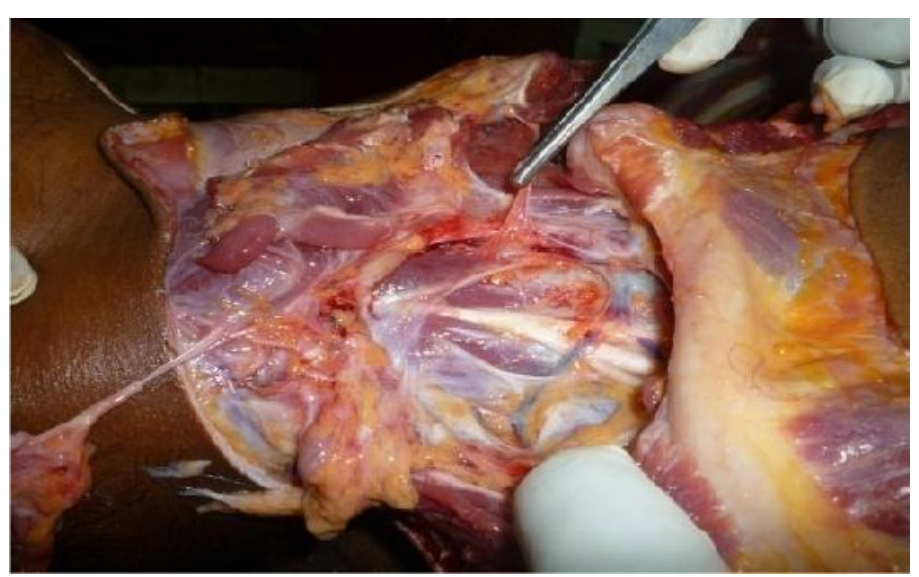

Fig.2. The ease of Neuro vascular hilum dissection is shown by the proximity of all the three structures namely External jugular vein, middle clavicular nerve and supraclavicular perforator artery.

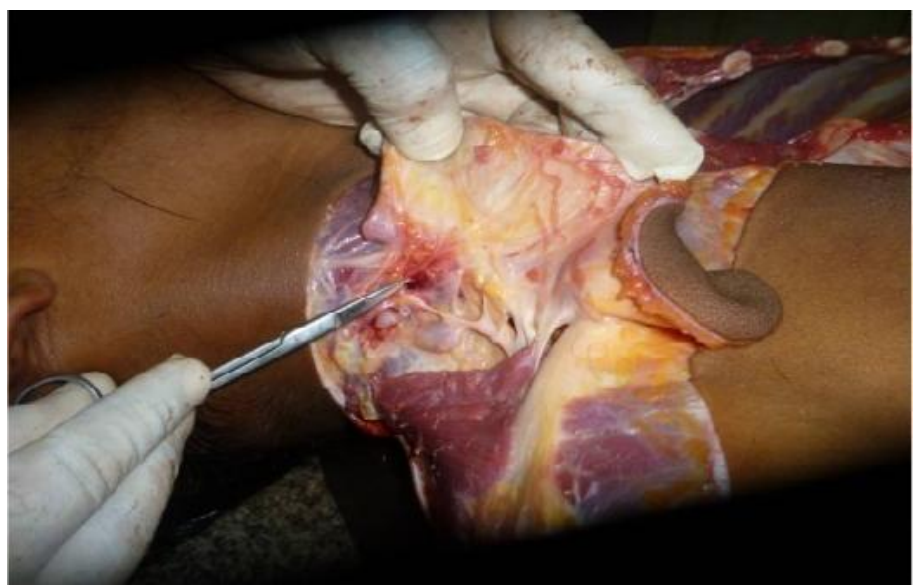

Fig.3.Pre operative picture of post burns scar contracture of neck
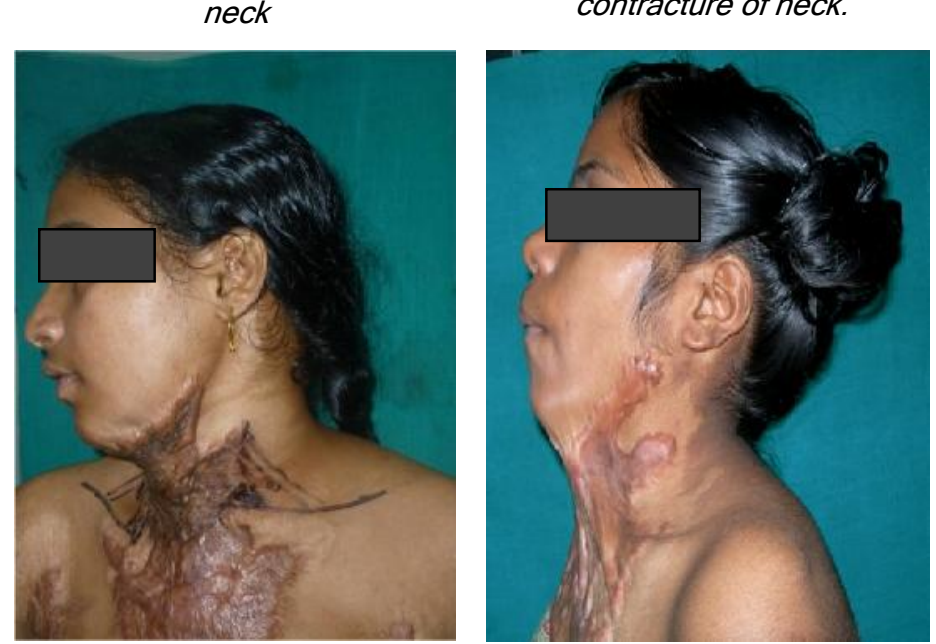

Fig.4. Pre operative picture of post burns scar contracture of neck.

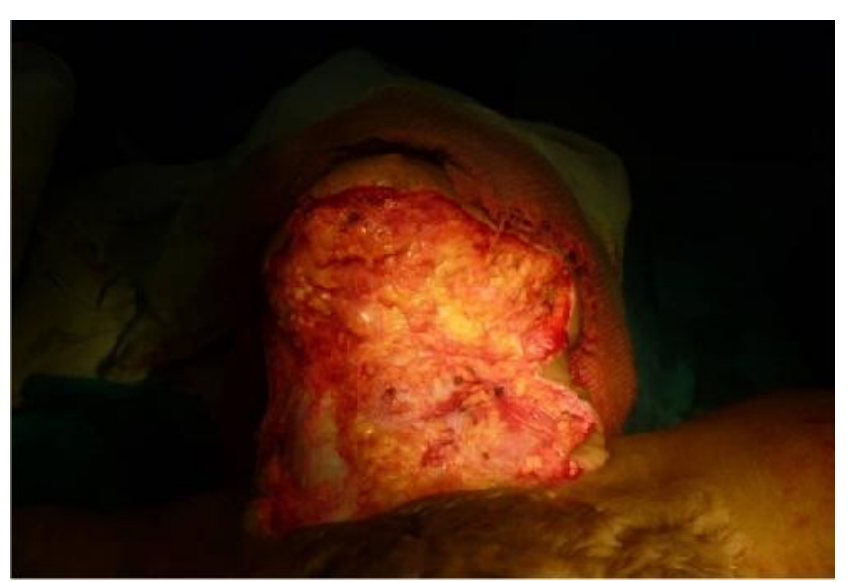

Fig.6. Intra operative SCAP flap marking

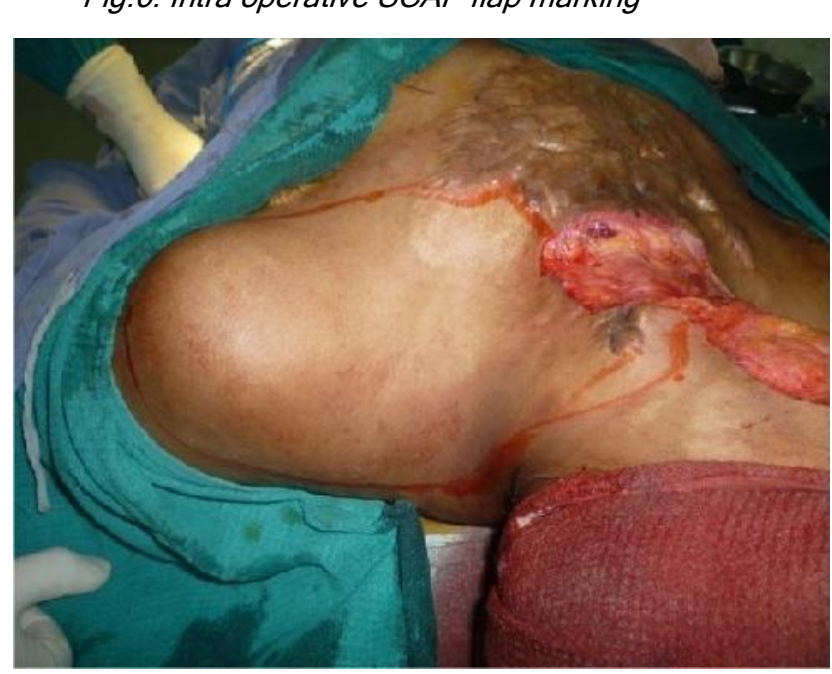

Fig.7. Flap harvested with pedicle

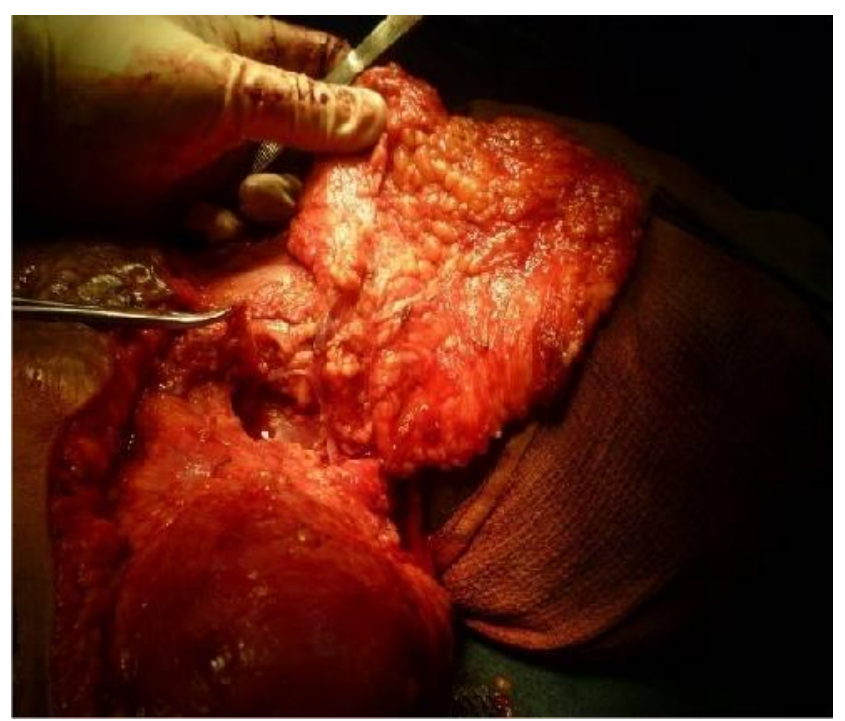

\section{Fig.5. Intraoperative scar excision}

Research article

CIndian Society for Education and Environment (iSee)

Vol. 5 No. 8 (August 2012) ISSN: 0974- 6846 
artery propeller (SCAP) enter and leave this flap (Fig.1 and 2).

\section{Significant findings of the anatomical study}

1. No venae commitants for SCAP, 2. SCAP Lateral to External jugular vein, 3. Proximity of middle supraclavicular nerve to the SCAP so that by dissection a Neurovascular hilum can be created for effective propeller movement. These significant variation contribute to the successful fabrication of sensate propeller SCAP flaps and these variations found in our study are not mentioned in the literature so far (Pallua, Norbert et al., 1997; Kneser et al., 2010).

\section{Clinical series}

From September 2010 to March 2012, 16 supraclavicular artery perforator flaps were used for various cases of mentosternal post burns scar contractures, their age ranging from 19 to 42 years, with 4 males and12 females. Four of cases were already treated with split skin grafting and has come with recurrence, twelve cases had deep 2 nd degree burns and 3rd degree burns healed with secondary intention, six cases had severe mentosternal contraction with severe lower lip ectropion, four cases had moderate contracture of neck with limitation of neck mobility. All patient had undergone surgery with their consent with fourteen cases orotracheal intubation after tumescent infiltration and release. One had bronchoscope aided intubation and one had blind nasotracheal awake intubation with mucosal anaesthesia (Fig.3 and 4).

\begin{tabular}{|l|l|}
\hline \multicolumn{2}{|c|}{ Table1. Clinical series } \\
\hline Cases & Size of flaps $(\mathrm{cms})$ \\
\hline C1 & $22 \times 20$ \\
\hline C2 & $33 \times 25$ \\
\hline C4 & $23 \times 23$ \\
\hline C5 & $27 \times 26$ \\
\hline C6 & $25 \times 21$ \\
\hline C7 & $22 \times 21$ \\
\hline C8 & $22 \times 19$ \\
\hline C9 & $24 \times 27$ \\
\hline C10 & $30 \times 27$ \\
\hline C12 & $31 \times 27$ \\
\hline C13 & $20 \times 21$ \\
\hline C14 & $23 \times 20$ \\
\hline C15 & $35 \times 27$ \\
\hline C16 & $21 \times 23$ \\
\hline Avera & $26 \times 22$ \\
\hline
\end{tabular}

Average operating time was one and half hour. All of them had medium thickness skin grafting for secondary defect, only unilateral flap was taken as the other donar side was found unsuitable for harvesting as it was scarred and also no delay and no supercharging was done in any of our cases despite three flaps exceeding $30 \mathrm{cms}$ in their greatest dimension but literature so far advises for supercharging whenever the greatest dimension exceeds $20 \mathrm{cms}$ (Pallua et al.,1997) . Transverse cervical artery was divided distal to perforator and External jugular vein just above clavicle. Sternocleido mastoid muscle clavicular head was divided to facilitate the propeller movement (Fig.5). The minimum flap size taken was $20 \times 21 \mathrm{cms}$, maximum flap size was $35 \times 27 \mathrm{cms}$. All the patients were counseled pre-operatively about the donor site and the possibility of stretching of the scar and hyperpigmentation post-operatively was explained (Table 1).

\section{Flap elevation}

After the complete excision of the scar in all post burn contracture and it was made sure the completion of excision by shoulder lifting test and rotating the head sideways. Then planning in reverse was done and the exact dimension of the flap decided. In all our cases the author found that SCAP readily recruited adjacent dynamic perforosomes like deltoid branch of thorocoacromial artery, the deltoid perforator of anterior and the posterior circumflex humeral system, trapezius musculocutaneous perforator from the deep branch of transverse cervical system. Using 10 mega Hertz Doppler the supraclavicular artery perforator detected (Ogawa et al., 2003). and transverse cervical system marked.The flap was marked in such a way that the dopplered perforator lies at the base of the flap with tongue shaped extension over the lesser supraclavicular fossa and sternoclavicular joint which will cover the pedicle following the propeller movement. The flap elevation was standardized by starting the elevation from the lateral (distal) aspect and progressing medially in a subfascial plane. The communicating perforators from the deltoid branch of the thoraco-acromial axis and the posterior circumflex humeral artery and the musculocutaneous perforator from deep branch of the transverse cervical system were sacrificed Near the point of exit of the supraclavicular vessels, the dissection was done in such a way that after ligating External jugular vein close to the clavicle recruiting middle supraclavicular nerve in the flap and including the fat and lymph nodes which is around supraclavicular artery. Some amount of retrograde dissection is also done from the transverse cervical system after it has been divided just before its bifurcation in front of levator scapulae. The pivot point for the flap can be shifted towards lesser supraclavicular fossa by dissection, as admissable by other dissected structures like External jugular vein and middle supra clavicular nerve (Fig.6).

It has been found by the author in his experience that the 270 degree rotation in the clock wise direction or anticlock wise direction to reach the defect has been found causing no congestion of the flap (depending upon the side of the donor site); on the other hand the shortest 90 degree rotation invariably causes congestion .In all cases External jugular vein is recruited in the flap with retrograde drainage, Thinning of the flap except around the pedicle is done in all cases. No cervicomental angle tacking stitches was done but cervicomental angle reforms spontaneously in all our cases (Mimoun et al.,1986). The planned tongue extension of the flap covers the pedicle of the flap. Medium thickness split thickness skin graft is used for covering secondary defect after ensuring hemostasis, Segmular drains were kept
Research article

CIndian Society for Education and Environment (iSee)
"Reconstruction of post burn scars" http://www.indjst.org
Balakrishnan \& Sivarajan Indian J.Sci.Technol. 
beneath the flap. Tie over dressings given for donar site to maximize graft bed adherence. Flap inset was given using 40 monocryl with cutting needle. If during the flap inset there is undue tension over the pedicle the clavicular head of the sternocleidomastoid muscle is divided close to the clavicle to facilitate tension less propeller migration of the pedicle (Fig.7).

Fig.8. Post operative picture (Front view)

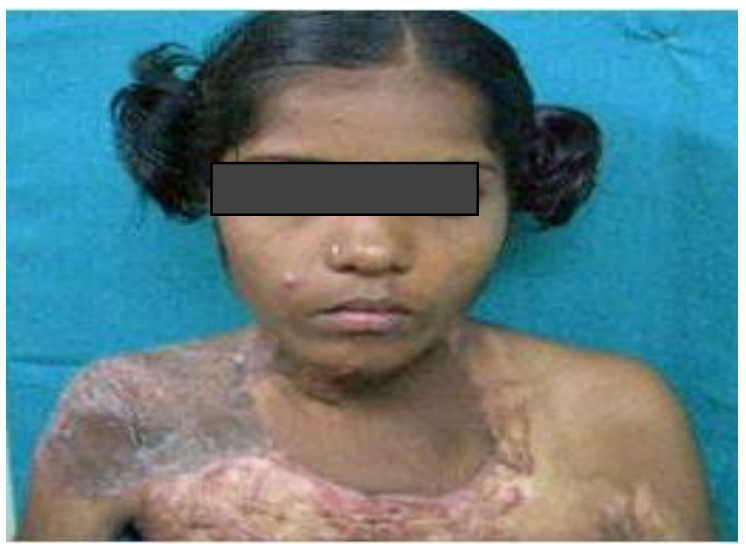

Fig.9.Post operative picture (Lateral view)

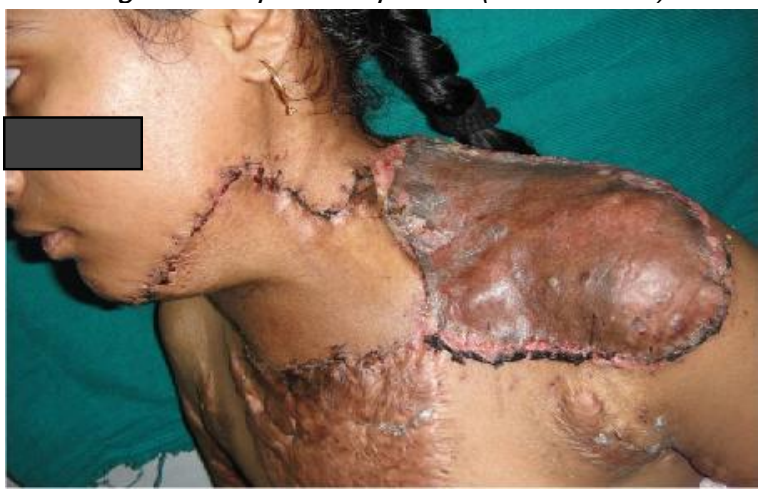

Fig. 10.Post operative picture (Lateral view).

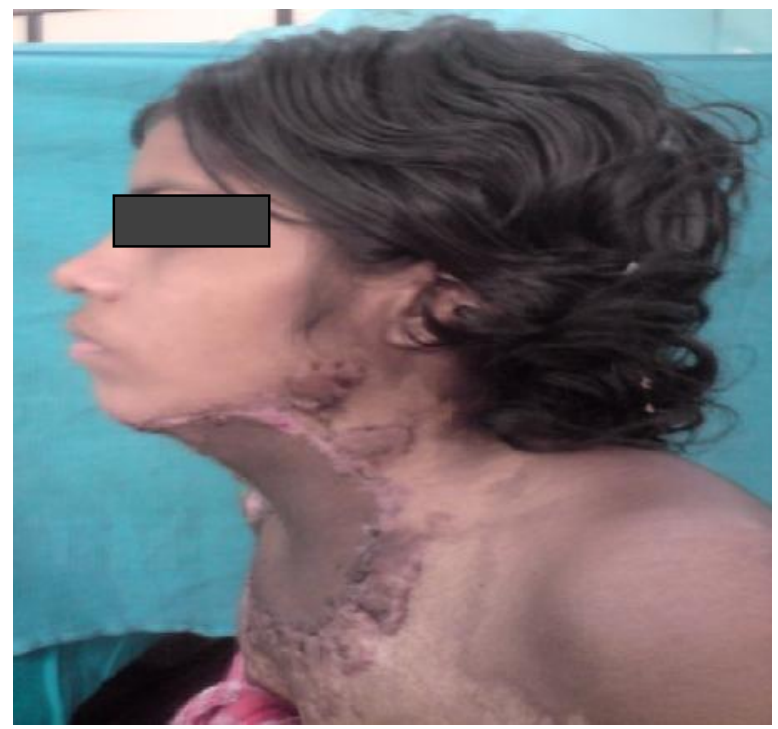

Post operative course:

Post operative blush reaction with bright red appearance was observed in all patients within 48 hours. Most of them disappeared with in 72hours (Villafane et al.,1986).Two point discrimination of $15 \mathrm{~mm}$ was noted in all quadrants of the flap in immediate post operative period .Without any quilting stitches described in few series, all patients had excellent cervico mental contour. Most of them said they can obscure the skin grafted region on the secondary defect with Indian type clothing .All had excellent mobility of neck with pain killer right from day one and none adopted cumbersome and awkward neck extension posture in the post op period. Segmuler drains used are removed at $48 \mathrm{hrs}$, no splints used in our cases.

Raising the flap as sensate not only helps in better proprioception in immediate postoperative period with absence of pain helps in these patients to mobilize the neck but also eschewed the hyper adrenergic drive so that the large dimension flaps are successfully done. As there was no venacommitantes seen with SCAP in all our clinical cases, we recruited EJV, which provided excellent venous drainage though in retrograde fashion which is also one of the reasons for large size flap survival without any need for supercharging. Even in one recurrent post burn contracture case, where External jugular vein was damaged there was a trivial vein found separate from the Supraclavicular artery. All patients were satisfied with aesthetic and functional results which are assessed by smiley chart.

\section{Complications}

Flap

Superficial epidermolysis of distal $5 \mathrm{~mm}$ observed in 36yr female asthmatic on steroid healed completely with conservative measures. Suture line abscess observed in one case on suture removal resulted in wound dehiscence and on secondary suturing healed well. No other complications related to flap noted in our series.

\section{Donor area}

Patchy Loss of graft observed in 3 cases at greater supraclavicular fossa near pedicle where regrafting done.

Except in one case with axillary contracture none had shoulder dysfunction or dyskinesia (Fig.8, 9, 10).

\section{Discussion}

The Anatomical study which has been done in the cadavers, had shown significant variations in relation to SCAP. So far in the literature SCAP has been described with venaecommitantes, (Cormack et al.,1984). In our series neither in cadaver nor in clinical cases SCAP was accompanied by venae commitantes. It is also described as a standard land mark that SCAP arises through sternocleidomastoid muscle, External jugular vein and The clavicular triangle but in all our cases including cadavers it has been found running lateral to External jugular vein . Recruiting middle supraclavicular nerve in the flap is not only anatomically facilitated but also in our
Research article

COIndian Society for Education and Environment (iSee)
"Reconstruction of post burn scars" http://www.indjst.org
Balakrishnan \& Sivarajan Indian J.Sci.Technol. 
clinical cases it was found that the point of origin from the erbs point of the sternomastoid muscle is in close proximity to External jugular vein and SCAP (Pallua et al.,1997).

Authors feel that very large flaps $35 \mathrm{cms}$ in greatest dimension was successfully done because of External jugular vein recruitment and intact middle supraclavicular nerve. External jugular vein recruitment provides good venous drainage in the retrograde fashion and also the middle supraclavicular nerves harvested along with the flap prevented immediate sympathectomy induced hyper adrenergic sensitivity of the arterioles. For these reasons no delay was done in any of our large size SCAP flaps. Authors have never done any supercharging in any of the clinical cases. It is also found that the blush reaction due to increased blood supply noticed in the immediate post operative period in all cases on an average up to 72 hours and this is similar to the staging of the flap during elevation itself. It is found that the middle supraclavicular nerve has wide overlapping over the medial Supraclavicular, lateral Supraclavicular and transversecervical cutaneous innervations areas. Modification of the flap design with tongue shaped extension over lesser supraclavicular fossa and sternoclavicular joint. Provided good coverage over the pedicle. With minimal analgesics all sixteen patients exhibited good range of neck mobility which is allowed $24 \mathrm{hrs}$ following surgery. It has been found in the follow up most of the patient $(90 \%) n=14$. felt that their appearance of face and neck improved and the donar site hyperpigmentation is of no concern to them as it can be easily obscured by Indian type of clothing. Only two patients complained about stretching of donar site scar over the shoulder. The follow up period ranges from six months to 24 months. There was excellent cervicomental angle reformation with no quilting stitch during the operation in all our cases (Mimoun et al.,1986).

\section{Conclusion}

Though many techniques are available for resurfacing of the post burns scar contracture of the neck after surgical release, this technically easier propeller sensate SCAP flap renders good functional and aesthetic results in the face and neck region with lasting results. With excellent colour texture ,thickness match with intact sensation SCAP flap is a versatile flap for reconstruction of post burns contracture of neck. This is better over sub dermal vascular network thin flap (Hyakusoku et al., 1994) harvested from the back in terms of colour and texture match. It is better than any key stone flaps harvested from the scapular and neck region again for good colour and texture match. For the same reason SCAP is the better choice over any microvascular transferred tissues. When SCAP harvested with middle supraclavicular nerve and External jugular vein as described by us, even large sized (more than $30 \mathrm{cms}$ by greatest dimension) flaps can be harvested with good success rate despite no delay or supercharging.

\section{References}

1. Achauer BM (1991) Neck reconstruction. In: Burn reconstruction. New York: Thieme.pp:79-86.

2. Pallua Norbert, Machens Hans-Guenther, Rennekampff Oliver, Becker Michael and Berger Alfred (1997) The fasciocutaneous supraclavicular artery island flap for releasing post burn mentosternal contractures. Plast Reconstr. Surg. 99 (7).

3. Kneser U, Beier JP, Dragu A, Arkudas A and Horch RE (2010) Transverse cervical artery perforator propeller flap for reconstruction of supraclavicular defects. J. Plast. Reconstr. Aesthet. Surg. 64(7), 952954.

4. Cormack GC and Lamberty BG (1983) The anatomical vascular basis of the axillary fasciocutaneous pedicle flap. Br.J. Plast. Surg.36, 425-427.

5. Cormack GC and Lamberty BG(1984) A classification of fascio-cutaneous flaps according to their patterns of vascularization. Br. J. Plast. Surg. 4, 37, 80-87.

6. Norbert Pallua and Erhan Demir (2009) The supraclavicular artery island flap in reconstructive facial surgery quality. Medical Publ., Inc., Plastic Surgery Pulse News. 2 (2), 1-2.

7. Hyakusoku H and Gao JH (1994) The "super-thin" flap. Br. J. Plast Surg.47, 457-464.

8. Ogawa R, Hyakusoku $H$ and Murakami M (2003) Color doppler ultrasonography in the planning of microvascular augmented super-thin (SVN: Subdermal vascular network) flaps. Plast. Reconstr. Surg.112, 822-888.

9. Mimoun M, Kirsch JM, Faivre JM and Baux S (1986) Rebuilding the cervico- mandibular angle: Correcting a deformity of neck burns. Burns Incl. Therm. Inj.12, 264-269.

10.Cormack CG and Lamberty BG (1994) The arterial anatomy of skin flaps. 2nd ed. New York: Churchill Livingstone.

11.Villafañe P. Stanley, Venkataramakrishnan V (1996) Breast reduction and carcinoma in situ. Br.J.Plastic Surgery. 49, (7), pp:499. 